\title{
A COST-EFFECTIVE VLSI ARCHITECTURE FOR HIGH-THROUGHPUT SEQUENTIAL DECODER*
}

\author{
Chen-Yi Lee \\ Dept. of Electronics Engineering, National Chiao Tung University, \\ 1001, University Road, Hsinchu 300, Taiwan, ROC \\ Tel: +886-35-731849; Email: cylee@cc.nctu.edu.tw
}

\begin{abstract}
This paper presents a new VLSI solution for high-speed digital communications based on long-constraint convolutional codes. The proposed VLSI architecture implements a modified sorter-based sequential decoding algorithm with an achievable maximum decoding rate of $25 \mathrm{Mbits} / \mathrm{s}$. Unlike its previous version based on shiftable content addressable memory (SCAM) only, path recording is now implemented on an emebedded SRAM module whose size is determined by path depth $(d)$ and survived nodes $(S)$. That is, both SCAM and SRAM are exploited to implement the sorter kernel. Results show that, for a $(2,1,7)$ code, both power consumption and silicon area can be improved by $50 \%$ on the average, making the new proposal very suitable for high-speed convolutional code applications.
\end{abstract}

\section{INTRODUCTION}

Convolutional coding with Viterbi decoding [1] is widely accepted as an efficient method to achieve a significant power gain on digital communication channels with low to moderate signal to noise ratios. Generally speaking, for information bit rates above $5 \mathrm{Mbits} / \mathrm{s}$, the decoder must be based on a fully parallel implementation of the Viterbi algorithm, which often demands one complete cycle in each clock interval and hence parallel ACS modules and path memory are requested. On the other hand, sequential decoding $[1,2]$ has low computation complexity in search of a correct path. However the decoding rate becomes lower because a correct path can only be identified after several trials on incorrect paths due to error bits. The principal sequential decoding algorithms are the Fano [2] and the stackbased [3]. Although these algorithms are useful for longer constraint length codes, they suffer from "buffer overflow" which derives from an inability to maintain uniform decoding rates while searching message sequences. Previously, we presented a dedicated memory structure [4] to speed up the decoding rate, where the buffer overflow is solved lately by shiftable content addressable memory (SCAM) [5]. Based

*WORK SUPPORTED BY THE NATIONAL SCIENCE COUNCIL OF TAIWAN, ROC UNDER GRANT NSC82-F-SP009-01. on such a newly developed high-speed data sorter [6], we can achieve a sorted sequence right after input samples are given. This technique provides us a very powerful hardware solutions for those applications which require massive sorting operations. However power consumption and silicon area are the two constraints for implementing longconstraint codes.

In this paper, we propose a new VLSI architecture to solve these two problems. In section 2, we first briefly describe the sorter-based sequential decoding algorithm, where features and complexity of the new algorithm will be highlighted. In section 3, we present a new VLSI solution to implement long-constraint sequential decoder. Comparison between the new solution and its previous version will also be given to show the improvements in both power consumption and silicon area. Finally some concluding remarks are drawn on the cost-effective solution.

\section{THE FAST SEQUENTIAL DECODING ALGORITHM}

In principle, the sequential decoding algorithm can be regarded as a set of sorting operations working on the survived nodes to identify a candidate node. And from this candidate node, further tracking can be performed until all input sequences are decoded. Figure 1 shows the decoding process for two cases. It can be found that the search of correct path is now formulated as a sorting procedure. To find such path, we have assigned different costs added to each node so that a local minimum can be located. However due to finite precision problem, we have to perform renormalization during the decoding process to ensure that each survived node does have the correct weight. In addition, a path recording technique was also developed to overcome back-tracking of correct bit sequences and to improve throughput rate. This is done by introducing two parameters: one is decoding status parameter which records the decoding depth from the root, the other is the path sequence parameter which records the decoded bits up to the survived node. This modified sequential decoding or named fast sequential decoding (FSD) algorithm was presented in [5].

The modified version FSD algorithm mainly consists of sorting and path recording kernels. The sorting kernel pro- 
vides storage space for the survived nodes which are sorted in ascending order. To reach high-throughput sorting results, we use delete-and-insert sort algorithm which has been mapped onto parallel comparison structure [6]. This implies that each storage node needs a comparator. As for path recording, a depth of up to $d$ is allocated for each survived node. This method keeps the decoded path and sends out the decoded bits until the depth of decoded path meets the specified value. Thus it totally needs $\left(d+\log _{2} d\right) \times S$ storage space, where $\log _{2} d$ and $S$ represent depth status and number of survived nodes respectively. The depth status parameter is used to track decoding process and output a decoded bit whenever an upperbound is encountered. The $S$ storage space is determined by constraint length to meet coding rate under a certain SNR ratio. Thus it should be noted that both $d$ and $S$ are highly dependent on the selected convolutional codes. More detailed discussions about these two parameters and their effects on coding gain of different codes can be found in $[5,7]$.

\section{THE VLSI ARCHITECTURE}

In this section, we present an efficient VLSI architecture for single-chip implementation of the developed algorithm. It is found that the constraint length is not limited by this proposed architecture and hence the proposed algorithm and architecture are very suitable for long constraint-length applications.

The proposed algorithm can be mapped onto an architecture as shown in Figure 2, which mainly consists of 6 units. The input buffer unit (IBU) contains the encoded sequences which have been arranged based on the coding rate defined in the corresponding encoder. It can be accessed by depth status parameter when error bits are detected. The weight calculation unit (WCU) uses the node with minimum weight as a basis and then calculates the new weight according to the costs generated by the cost generation unit (CGU). The cost is obtained by pattern matching and then produces a set of costs. The weight sorting unit (WSU) is used to store the weights of the survived nodes. The path recording unit (PRU) is provided to produce the correct bit sequence when target depth is reached and, in the mean time, to give a label indicating the order of decoded bits. Detailed structure of this unit is given in Figure 3 which mainly consists of path memory, shifter, incrementer and detector.

Implementation results [8] show that SCAM realization of PRU and WSU occupies most of the chip area and power dissipation because of simultaneous shift operations. To solve these two problems, we have recently combined SCAM and SRAM as shown in Figure 4. Here we use address as an indicator to link to path sequences which are now stored in SRAM. These addresses and weights of survived nodes are stored in the SCAM. During the sorting process, only the weight from the first node is read ont and added with two costs. Then the two generated weights are simultaneously compared to the other weights. Once this step is done, two addresses from the SCAM are derived and connected to SRAM. Both addresses are used to update the path information stored in the SRAM, such as path sequence, decoded status. These paths are the " 0 " path and " 1 " path which can simultaneously be stored in the SRAM. However, the correct path from the first address is always activated to provide address to input buffer, from which new input sequences can be decoded or error bits can be recovered. Note that in practical realization, this is the critical path. Therefore pre-fetching strategy is exploited here to speed up decoding process. This is done by selecting the contents of the first node for next address to the input buffer. For most of the time, this method does apply to most of the input patterns since error bits are only very small part of the input sequences. If the addresses are not directly obtained from the first node, the address should be taken from the second node. This implies that due to error bits, both new weights are larger than that of the second node. In other words, the address for input buffer can be obtained either from the first node or the second node depending on the input patterns. Only when error bits are detected, will the decoding rate becomes slower because more time is needed to perform read/write operations on the SRAM. Currently the critical path is limited to $20 \mathrm{~ns}$ for $0.8 \mu \mathrm{m}$ CMOS double metal process.

Operations between SRAM and SCAM are described as follows. Initially addresses and weights are assigned to each storage element of SCAM. Both left and right elements provide two addresses to SRAM so that path information can be updated. This strategy is derived because the left element always stores the candidate node with minimum weight, where it has to be traced further; and the right element always stores the candidate node with maximum weight, implying that it's no long a possible candidate and can be overwritten by other newly generated nodes. Each time when a new node is created, its address for the path information should be updated in the SCAM. As shown in Figure 5(a), both addresses from the SCAM are connected to SRAM to update path information. However a pre-fetched path register is inserted to speed up the updating process. That is when no error bits are detected, only one write operation is requested for the SRAM; however when error bits are encountered, two write and one read operations are requested for the SRAM. Figure 5(b) shows the basic cell of the multi-port SRAM which can be written from two different address buses.

Thus for each survived node, only $\left(\log _{2} W+\log _{2} S\right)$ is needed in SCAM, instead of $\left(\log _{2} W+\log _{2} d+d\right)$. For each survived node, we need $\left(\log _{2} d+d\right)$ storage space in SRAM. At first sight, it is found that more storage space, i.e. $\log _{2} S$, is needed in the proposed architecture. However physical design shows that both area and power can be reduced by $50 \%$ on the average. For more long constraint code applications, the gain in power dissipation and area will become more evident because many shift operations in the SCAM 
can be replaced by read/write operations in the SRAM. In addition, the basic SRAM cell is only about half size of the SCAM cell. Table 1 lists the implementation data compared to its previous SCAM version for a $(2,1,7)$ code.

To run this architecture, it is necessary to initialize the SCAM so that addresses and weights are placed appropriately. Once this procedure is done, the decoding process can be activated. Our simulation results show that the performance from this architecture can compete those results obtained from maximum likelihood decoder. Thus with this architecture, not only high-speed and programmability can be achieved but also less power consumption and silicon area can be obtained for long-constraint codes, making it very suitable for practical applications.

\section{CONCLUSION}

In this paper, we have presented a new VLSI architecture for sorter-based sequential decoding algorithm. The use of both sorting and path recording techniques not only solves the low-throughput problem but also provides a solution for long constraint convolutional code designs when mapped onto SCAM and SRAM architecture. Thus a cost-effective solution for high-speed convolutional codes can be achieved. We are currently developing a radix-4 FSD algorithm and architecture in order to enhance decoding rate for highspeed networking applications.

Acknowledgement: The authors would like to thank their colleagues within the SI2 group of National Chiao Tung University for many fruitful suggestions and discussions. The MPC support from NSC/CIC for the demonstrator chip is also acknowledged.

\section{REFERENCES}

[1] A.J. Viterbi and J.K. Omura, "Principles of Digital Communication and Coding", McGraw-Hill, 1979.

[2] R.M. Fano, "A Heuristic Discussion of Probabilistic Decoding", IEEE Trans. Inform. Theory, Vol. IT-9, pp. 64-73, 1963.

[3] F. Jelinek, "Fast Sequential Decoding Algorithm Using a Stack", IBM J. Res. Develop., pp. 675-685, Nov. 1969.

[4] C.Y. Lee, F. Catthoor, and H. De Man, "Breaking the Bottleneck of Sequential Decoding for High-Speed Digital Communication", Proc. of ICASSP, Toronto, May 1991.

[5] C.Y. Lee, W.W. Yang, and L.F. Jeng, "A Fast Sequential Decoding Algorithm Based On Dynamic Searching Strategy", IEE Proc.-I: Communications, Vol. 141, No. 5, October 1994.

[6] C.Y. Lee, J.M. Tsai, and P.W. Hsieh, "High-Speed Median Filter Designs Using Shiftable Content Address Memory", IEEE Trans. on Circuits and Systems for Video Technology, Vol 4, No. 6, Dec. 1994.

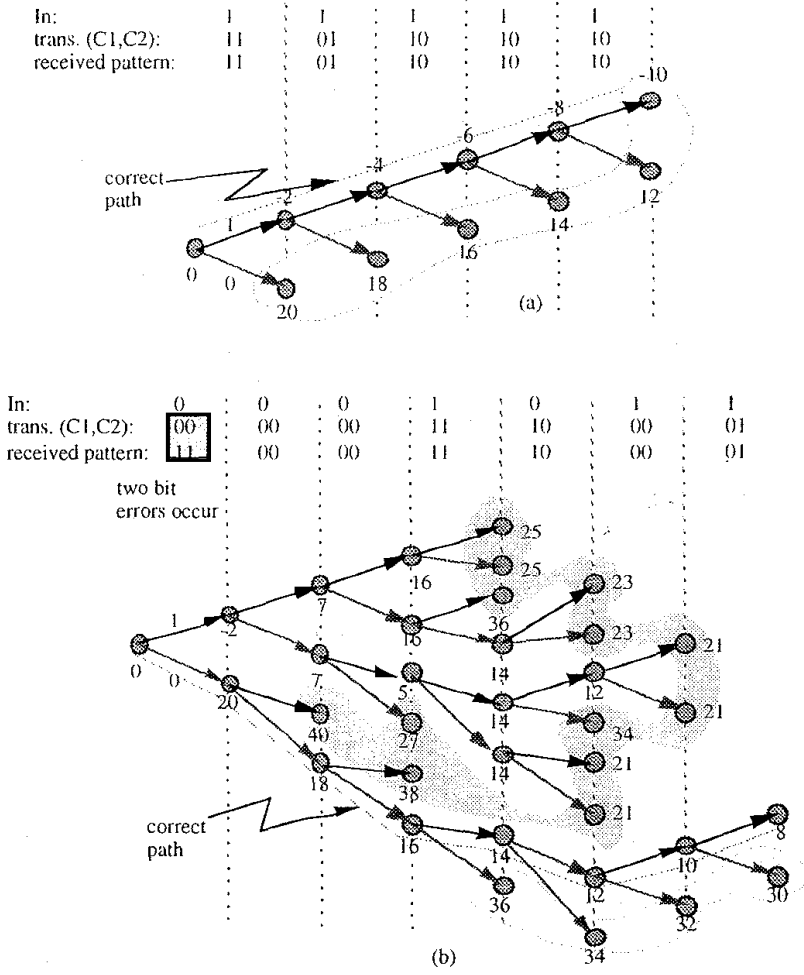

Fig.1. Illustration of decoding process based on sorter-based sequential decoding algorithm.

[7] C.Y. Lee and W.W. Yang, "A Fast Sequential Decoding Algorithm Based On Dynamic Searching Strategy", IEE Proc.-I: Communications, Vol. 141, No. 5, October 1994.

[8] W.W. Yang, "Fast Sequential Decoding Based on HighSpeed Sorter", NCTU/DEE Master Thesis, June 1994.

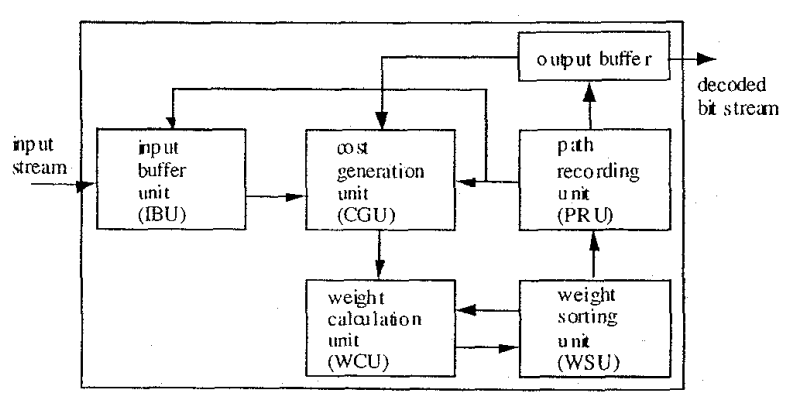

Fig.2. Block diagram of the sorter-based sequential decoder chip. 


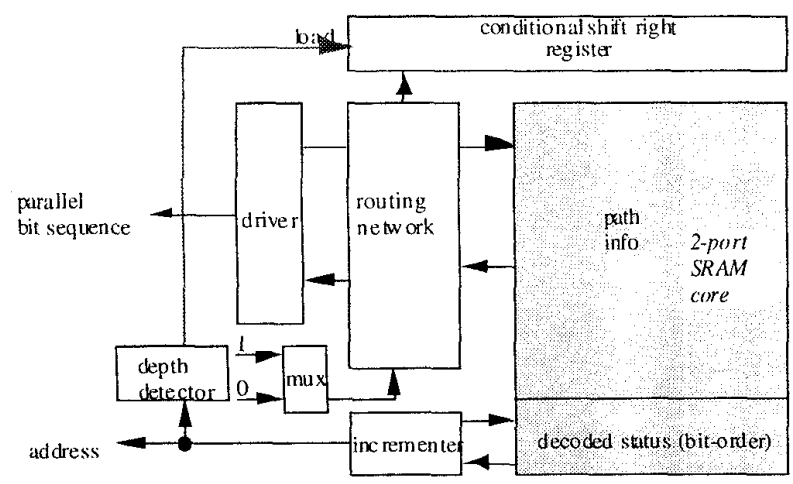

Fig.3. Detailed structure of the path recording unit.
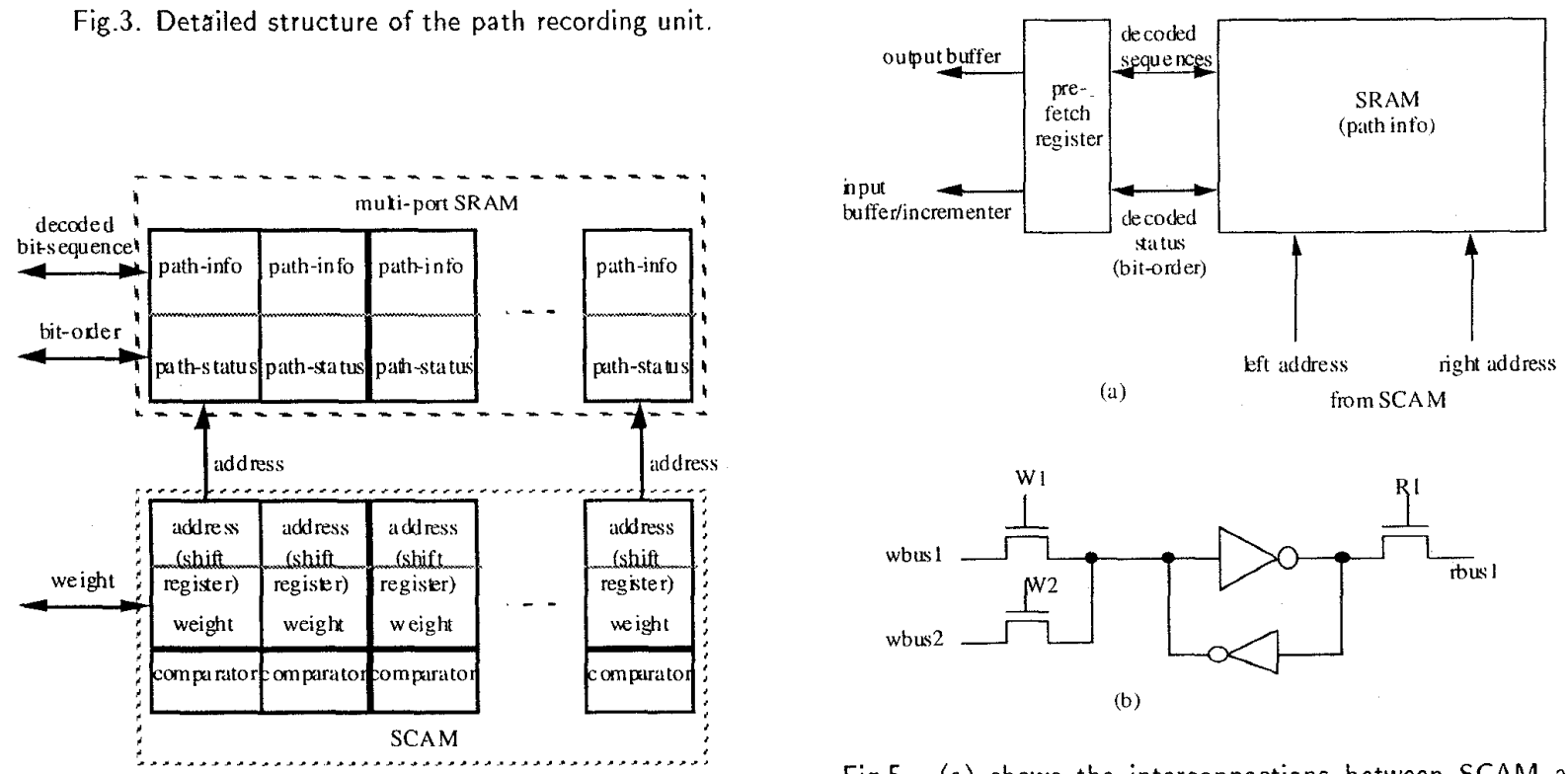

(b)

Fig.4. Combining SCAM and SRAM to implement high-speed sorting and path recording.

Fig.5. (a) shows the interconnections between SCAM and SRAM and (b) shows the leaf cell for the multi-port SRAM.

Table 1.1 Comparison between SCAM-based and SCAM/SRAM-based architecture for the sorter-based sequential decoder.

\begin{tabular}{|c|c|c|}
\hline \hline style & SCAM & combined both SCAM and SRAM \\
\hline storage space & $\log _{2}(W \times d)+d$ & $\left(\log _{2}(W \times S)_{S C A M}+\left(\log _{2} d+d\right)_{S R A M}\right.$ \\
\hline power consumption & $0.8 \mathrm{~W}$ & $0.4 \mathrm{~W}$ \\
\hline area & $5.8 \mathrm{~mm}^{2}$ & $2.8 \mathrm{~mm}^{2}$ \\
\hline improvement & - & $50 \%$ \\
\hline
\end{tabular}

Note: the above data is achieved for $(2,1,7)$ code design. 